\title{
Preparation of Gold Nanoisland Arrays from Layer-by-Layer Assembled Nanoparticle Multilayer Films
}

\author{
Hyung Y. Choi, ${ }^{\dagger}$ Michael S. Guerrero, ${ }^{\ddagger}$ Michael Aquino, ${ }^{\dagger, \neq}$ Chuhee Kwon, ${ }^{* \neq}$ and Young-Seok Shon ${ }^{*, \dagger}$ \\ ${ }^{\dagger}$ Department of Chemistry and Biochemistry, California State University Long Beach, Long Beach, CA 90840, USA \\ *Department of Physics, California State University Long Beach, Long Beach, CA 90840, USA. "E-mail: yshon@csulb.edu \\ Received September 4, 2009, Accepted November 9, 2009
}

\begin{abstract}
This article introduces a facile nanoparticle self-assembly/annealing method for the preparation of nanoisland films. First, nanoparticle-polymer multilayer films are prepared with layer-by-layer assembly. Nanoparticle multilayer films are then annealed at $\sim 500^{\circ} \mathrm{C}$ in air to evaporate organic matters from the films. During the annealing process, the nanoparticles on the solid surface undergo nucleation and coalescence, resulting in the formation of nanostructured gold island arrays. By controlling the overall thickness (number of layers) of nanoparticle multilayer films, nanoisland films with various island density and different average sizes are obtained. The surface property of gold nanoisland films is further controlled by the self-assembly of alkanethiols, which results in an increased surface hydrophobicity of the films. The structure and characteristics of these nanoisland film arrays are found to be quite comparable to those of nanoisland films prepared by vacuum evaporation method. However, this self-assembly/annealing protocol is simple and requires only common laboratory supplies and equipment for the entire preparation process.
\end{abstract}

Key Words: Nanoislands, Nanoparticles, Self-Assembly, Plasmonic Surfaces, Gold

\section{Introduction}

The detection of molecular interactions using a simple and small nanostructured array has drawn an intense interest, because it offers several benefits such as the use of small analyte volumes and the rapid response read-outs. ${ }^{1,2}$ Especially, the nanostructure-based plasmonics have recently attracted a great deal of attention in miniaturized optical devices and sensors as well as in medical diagnostics. ${ }^{3}$

Surface plasmon resonance (SPR), which is one of the most popular choices as a plasmonic sensing system, is a label-free technique that can provide data on adsorption and/or desorption events that occur at a metal/dielectric interface. ${ }^{4-6}$ For more than 20 years, SPR sensors, which are based on copper, gold, or silver planar films, have been used as refractive index-based sensors to detect analyte binding. The preponderance of optical sensing applications based on SPR is that it must be measured in an attenuated total reflection mode. ${ }^{3,7}$ In addition, SPR sensors require a quite large area $(10 \mu \mathrm{m} \times 10 \mu \mathrm{m})$ for sensing experiments.

Recently, localized surface plasmon resonance (LSPR)-based nanosensors are developed to complement the existing SPR sensors. ${ }^{8-22}$ In LSPR sensing, the spot size can be minimized to a small number of individual sensing elements with an in-plane width of less than $\sim 100 \mathrm{~nm}$. Therefore, LSPR-based nanosensors can be extremely simple, small, and light. In addition, the LSPR nanosensors can be used in both transmission mode and reflection mode. ${ }^{8-22}$ When UV-vis extinction spectroscopy in transmission geometry is used for sensing, it is known as transmission localized surface plasmon resonance (T-LSPR) sensing. The major advantage of T-LSPR sensing over conventional SPR sensing lies in the low-cost samples and simple experimental setup, which can be reduced to measurements at a single wavelength in a transmission configuration.

The T-LSPR nanosensor arrays based on small gold or silver nanostructures have been extensively studied by Rubinstein, Van Duyne, and others. ${ }^{8-31}$ Rubinstein's method involves a preparation of ultrathin (less than $10 \mathrm{~nm}$ ) semi-transparent gold island films by vacuum evaporation onto inert transparent substrates. ${ }^{15-22}$ Van Duyne's nanosphere lithographic techniques involve the fabrication of arrays of triangular silver nanoparticles in the interstices between the elements of the nanosphere deposition mask. ${ }^{8-14}$ The removal of the deposition mask following thermal deposition, pulsed laser deposition, or e-beam deposition of metal leaves triangular nanoisland arrays on the solid substrates. Changes in the gold or silver surface plasmon (SP) extinction induced by the binding of various analytes to the island surface or to a molecular layer on the island surface can be monitored using transmission UV-vis spectroscopy.

This paper describes our attempts to develop a new method that can replace or complement the previously reported processes of lithography and thermal vacuum evaporation for the nanoisland film preparation. Our proposed method is based on a facile nanoparticle self-assembly/annealing method, which is simple and requires only common laboratory supplies and equipment for the entire process. In addition, this method allows us to change the parameters (e.g. island size) that control the sensitivity of gold nanoisland films by simply altering the conditions of nanoparticle multilayer film preparation.

Previously, preparation of thin metal films generated by thermolysis of nanoparticle multilayer films has been demonstrated by the Murray group. ${ }^{32}$ In this method, multilayer films of carboxylate-functionalized alkanethiolate-protected nanoparticles were assembled using $\mathrm{Cu}^{2+}$ coordinative bridges. The nanoparticle multilayers were thermally decomposed at moderate temperature $\left(<350{ }^{\circ} \mathrm{C}\right)$ to produce films of the core metal. The thiolate ligands surrounding the nanoparticle cores escaped as volatile disulfides. This resulted in the formation of solid, continuous metal films rather than nanostructured metal films. The 
formation of continuous metal films in this study was resulted from the use of relatively thick nanoparticle multilayer films and the presence of the highly mobile linkers $\left(\mathrm{Cu}^{2+}\right)$.

Our attempt is based on the preparation of gold nanoisland arrays from nanoparticle multilayer films with a controlled number of nanoparticle layers, so that there can only be enough concentration of gold to form discontinuous films. Organic linkers that can resist high mobility of nanoparticles on solid surface are also used to help facilitate the formation of nanoisland-like structures. In specific, self-assembly/annealing of nanoparticle multilayer films containing small gold nanoparticles $(\sim 2.2 \mathrm{~nm})$ and polymer linkers is attempted for the formation of isolated nanodomains on glass substrates.

\section{Experimental Section}

Materials. The following materials were purchased from the indicated suppliers and used as received: Hydrogen tetrachloroaurate $\left(\mathrm{HAuCl}_{4} \cdot 3 \mathrm{H}_{2} \mathrm{O}\right)$, tetraoctylammonium bromide, toluene, sodium borohydride $\left(\mathrm{NaBH}_{4}\right)$, ethyl alcohol, acetone, tetrahydrofuran (THF), glass microscope slides, and 3-mercaptopropyl trimethoxysilane (MPTS) were purchased from Fisher Scientific. A 1-pentadecanethiol, 1-dodecanethiol, 1-nonanethiol, 1-hexanethiol, 11-mercapto-1-undecanoic acid, and poly (allylamine hydrochloride) (PAH; MW ca. 70,000) were purchased from Aldrich. Water was purified by Barnstead NANOpure Diamond ion exchange resins purification unit.

Synthesis of COOH-Functionalized Gold Nanoparticles. The $\mathrm{COOH}$-functionalized gold nanoparticles (MUA-NPs: average molecular formula of $\mathrm{Au}_{314} \mathrm{~L}_{91}$ ) used for the film preparation were synthesized by the following methods. ${ }^{33-35}$ Hexanethiolateprotected gold nanoparticles were first synthesized using a convenient two-phase synthesis known as the Schiffrin reaction. Briefly, $\mathrm{AuCl}_{4}^{-}$was transferred to toluene using tetraoctylammonium bromide as the phase-transfer reagent. The addition of hexanethiol $\left(2 / 1\right.$ mole ratio to $\left.\mathrm{HAuCl}_{4}\right)$ to organic-phase $\mathrm{AuCl}_{4}{ }^{-}$ followed by the reduction with $\mathrm{NaBH}_{4}$ generated hexanethiolate-protected gold nanoparticles with average core size of $\sim 2.2$ $\mathrm{nm}$. The incorporation of $\mathrm{COOH}$-functional groups to the monolayer involved a modification of the hexanethiolate-protected gold nanoparticles by ligand-replacement. ${ }^{34,35}$ In the exchange reaction, the incoming 11-mercaptoundecanoic acid (MUA) ligands replaced the hexanethiolate ligands on nanoparticles in THF by an associative reaction. The relative concentration of incoming ligands was carefully controlled for the synthesis of nanoparticles to have $\sim 20 \%$ of $\mathrm{COOH}$ functional groups in the monolayers. The ${ }^{1} \mathrm{H}-\mathrm{NMR}$ spectroscopy was used to determine the extent of the ligand exchange.

Functionalization of Glass Slides. The silanization procedure started with glass microscope slides that were cleaned in a "piränha" solution $\left(3 / 1 \mathrm{H}_{2} \mathrm{SO}_{4} / \mathrm{H}_{2} \mathrm{O}_{2}\right)$, rinsed thoroughly with distilled water, and placed in a $100 \mathrm{~mL}$ isopropyl alcohol solution containing $1 \mathrm{~mL}$ of MPTS and $1 \mathrm{~mL}$ of nanopure water. ${ }^{35}$ (Caution: Pirahha solution reacts violently with organic materials and should be handled with extreme care.) After heating for $30 \mathrm{~min}$, the glass slides were rinsed with ethanol, blown dry with $\mathrm{N}_{2}$, and allowed to dry in a $100{ }^{\circ} \mathrm{C}$ oven for at least $30 \mathrm{~min}$. These previous processes (heating, washing, and drying) were repeated at least two more times. The prepared glass slides were stored in a dry cabinet for future use.

Preparation of Gold Nanoparticle Multilayers. For multilayers containing polymer linkers, $10 \mathrm{mg}$ of PAH was dissolved in $10 \mathrm{~mL}$ of nanopure water yielding $c a .14 \mu \mathrm{M}$ solution concentration. The $c a$. $30 \mu \mathrm{MNP}$ solutions used in the buildup of multilayers were made by dissolving $10 \mathrm{mg}$ of MUA-NPs in $10 \mathrm{~mL}$ of pure ethanol. To build the gold nanoparticle multilayers onto glass slides, the MPTS-functionalized glass slides were placed in the ethanol solution containing MUA-NPs $(\sim 2.2 \pm 0.8 \mathrm{~nm}$ in diameter) for 24 hours. ${ }^{35}$ Then, the slides were alternately placed in the aqueous solution containing PAH and in the nanoparticle solution for ten minutes each to build the second layer of the gold nanoparticle films. This last procedure was repeated three or five more times to build five or eight layers, respectively, of the gold nanoparticle multilayer films. ${ }^{35} \mathrm{UV}$-vis spectroscopy was used to monitor the multilayer film formations.

Themal Annealing of Nanoparticle Multilayer Films. Nanoparticle multilayer films were heated in a Barnstead Thermolyne 1300 furnace under air for one hour and characterized by monitoring the changes in the absorbance of nanoparticle films by UV-vis spectrophotometer. The controlled temperature was set at $100{ }^{\circ} \mathrm{C}, 200{ }^{\circ} \mathrm{C}, 300{ }^{\circ} \mathrm{C}, 400{ }^{\circ} \mathrm{C}$, or $500{ }^{\circ} \mathrm{C}$ each within \pm 5 ${ }^{\circ} \mathrm{C}$. The annealed slides were left to cool in air to room temperature and stored immediately in a dry cabinet.

Preparation of Biotin-Functionalized Gold Nanoisland Films. Each slide was placed overnight in $1.0 \mathrm{mM}$ MUA solution $(2.2$ $\mathrm{mg}$ of MUA in $10 \mathrm{~mL}$ ethanol). The slide was washed with ethanol and placed in a $10 \mathrm{mM}$ DCC solution $(21 \mathrm{mg}$ DCC in $10 \mathrm{~mL}$ ethanol) for 2-3 hours. The slide was washed with ethanol and then placed in a solution containing $0.1 \mathrm{M}$ ethylene diamine ( $0.067 \mathrm{~mL}$ ethylene diamine and $10 \mathrm{~mL}$ ethanol) for 2-3 hours. Then the slide was washed with ethanol and placed in a solution containing 1.0 mM Biotin and 2.0 mM DCC (4.4 mg DCC, 2.5 $\mathrm{mg}$ biotin, and $10 \mathrm{~mL}$ ethanol) for 2-3 hours.

T-LSPR Sensing of Streptavidin. The slide was placed in a cell with phosphate-buffered saline (PBS) solution and measured the UV-reading of absorbance $v s$. wavelength using the UV-2450 UV-vis spectrophotometer. This was noted as a 0 minute measurement. The PBS solution was replaced with the solution containing $100 \mu \mathrm{g} / \mathrm{mL}$ or $1 \mu \mathrm{g} / \mathrm{mL}$ of streptavidin in PBS. A UV reading was taken every 2 minutes after the addition of streptavidin solution.

Measurements. Transmission UV-vis spectra of nanoparticle multilayer films and nanoisland films were acquired on a Shimazu UV-2450 UV-vis spectrophotometer with a film (slide) holder. A baseline correction procedure (the spectrum of a standard glass slide was taken as baseline) was executed prior to each measurement session.

Transmission electron microscopy (TEM) images of nanoparticles were obtained with a JEOL 1200 EX II electron microscope operating at $120 \mathrm{keV}$. Samples were prepared for TEM by casting a single drop of a toluene solution onto standard carbon-coated ( $80-100 \AA$ ) Formvar film on copper grids (200 mesh) and drying in air for at least $30 \mathrm{~min}$. Several regions were imaged at 200,000X. Size distributions of the gold cores were obtained from digitized photographic enlargements with Scion Image Beta Release 2. 
The TGA analysis was conducted on a SDT Q600 thermal analysis instrument using an ultrahigh purity (UHP) nitrogen atmosphere (flow rate of $100 \mathrm{~mL} / \mathrm{min}$ ), with heating from room temperature to $600{ }^{\circ} \mathrm{C}$ at a heating rate of $20{ }^{\circ} \mathrm{C} / \mathrm{min}$.

Contact angles of $\mathrm{H}_{2} \mathrm{O}$ were measured at room temperature and an ambient relative humidity using KSV model CAM 101 contact angle goniometer. The contacting liquids were dispensed and withdrawn at the slowest possible speed ( $c a .1 \mu \mathrm{L} / \mathrm{s}$ ). The data was collected and averaged over three separate slides using 3 spots per slide for each type of films.

Atomic force microscopy (AFM) images were acquired in an ambient condition with Nanoscope IIIa Multimode scanning probe microscope (Digital Instruments, Santa Barbara, CA) using tapping mode. A silicon probe with the EV scanning head was employed. The nanoisland films were imaged with no further sample preparations. Most of the images were obtained using Tap 150Al-G tips from Budget Sensors. Scanning parameters varied with individual tips and samples, but typical ranges were as follows: tapping frequency of $150 \mathrm{kHz}$ and scan rate of 1 - $3 \mathrm{~Hz}$. The grain size distribution was obtained from the AFM images using watershed segmentation in ImageJ.

\section{Results and Discussion}

Preparation and Annealing of Gold Nanoparticle Multilayer Films. Recently, we have reported the layer-by-layer (LbL) assembly and the stability evaluation of nanoparticle hybrid multilayer films on solid surfaces. ${ }^{35}$ Self-assembly of nanoparticle multilayer films was based on an interaction between carboxylic acid $(\mathrm{COOH})$-functionalized nanoparticles and linkers. ${ }^{35-39}$ Previously, the linkers such as metal cations, ionic polymers, and dendrimers were used for the preparation of various nanoparticle hybrid multilayer films.

It was known that the solid-state annealing of nanoparticles at relatively low temperatures $\left(100-250{ }^{\circ} \mathrm{C}\right)$ results in the nucleation and aggregation of nanoparticles. ${ }^{40-42}$ Controlling the degree of nucleation during this annealing process and the concentration of gold nanoparticles in the films is critical in terms of the preparation of gold nanoisland films rather than the formation of random metallic gold films. It is likely that a fast aggregation of particles will cause the formation of thin gold films or large gold aggregates, depending on the concentration of gold nanoparticles. In comparison, a slow aggregation will likely retain gaps between the groups of nanoparticles and result in the formation of small gold island domains.

Our previous studies examined the nucleation and aggregation tendency of nanoparticle hybrid multilayer films with different linkers by annealing the films at $\sim 100{ }^{\circ} \mathrm{C}$. 35 The change in absorbance intensity and the shift in SP bands in UV-vis spectra are related to internanoparticle spatial, particle aggregation, and surrounding medium properties. Spectroscopic investigations before and after heating treatments at $\sim 100{ }^{\circ} \mathrm{C}$ indicated that the thermally induced structural evolution of nanoparticle multilayer films was dependent upon the chemical nature of linkers. Nanoparticle-polymer multilayer films among various nanoparticle linker systems exhibited a high stability against a heating treatment at $\sim 100{ }^{\circ} \mathrm{C}$. This suggests that ionic polymers prevent a fast aggregation of nanoparticles on the surface more effi-

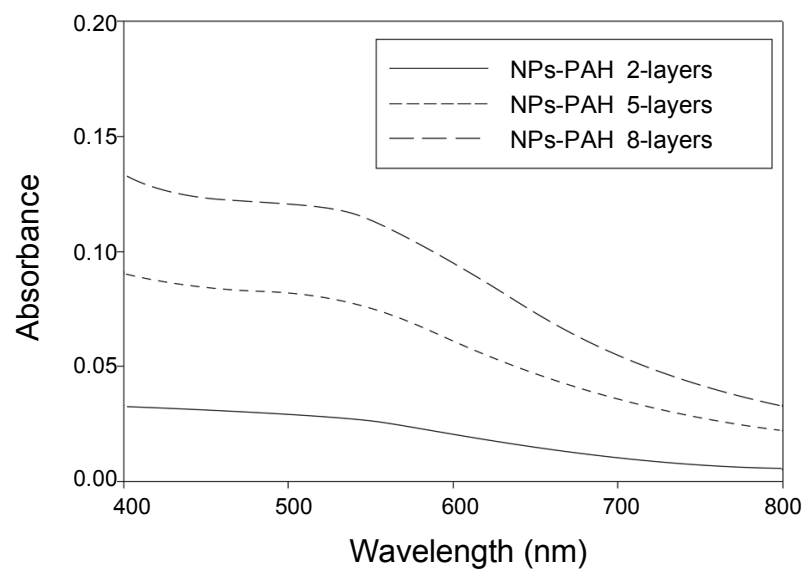

Figure 1. UV-vis spectra of nanoparticle multilayer films with 2-, 5-, and 8-layer nanoparticle assemblies.

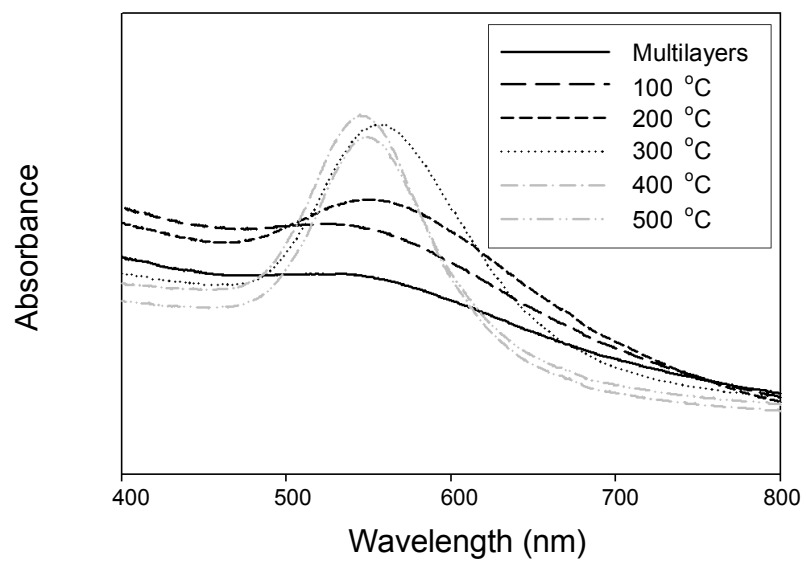

Figure 2. UV-vis spectra of nanoparticle multilayer assemblies (5nanoparticle layers) before and after annealing at the temperature ranging from 100 to $500{ }^{\circ} \mathrm{C}$.

ciently. The multiple linkages formed between polymer linkers and nanoparticles likely cause the diffusion of nanoparticles in the polymeric matrix more difficult. Since poly(allylamine hydrochloride) (PAH) is also an inexpensive and accessible industrial reagent, nanoparticle multilayer films based on $\mathrm{PAH}$ linkers were chosen as a target precursor for nanoisland films.

The LbL films of gold nanoparticles-PAH linkers (Au NPs$\mathrm{PAH}$ ) were grown on the surface of reactive glass substrates as described in Experimental Section. UV-vis spectra of gold nanoparticle films are known to exhibit surface plasmon (SP) band of gold at $\sim 525 \mathrm{~nm}$. This band gradually becomes more evident as successive layers are added to the films. Figure 1 shows the UV-vis spectra of Au NPs-PAH multilayer films containing 2, 5 , or 8 layers of gold nanoparticles on glass substrates. Nanoparticle multilayer films with a controlled number of nanoparticle layers were prepared for the information regarding the concentration of gold nanoparticles that can generate discontinuous films. The wavelength of the SP band of gold showed a subtle increase and broadening toward the longer wavelength region from $\sim 525 \mathrm{~nm}$ to $\sim 540 \mathrm{~nm}$ with an increasing film thickness (increasing number of nanoparticle layers). These slight red-shifts are the result of the smaller interparticle distance 


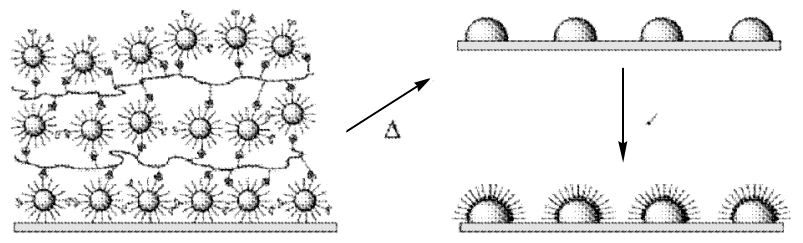

Scheme 1. Preparation of gold nanoisland films from nanoparticlepolymer multilayer films and formation of self-assembled monolayers.

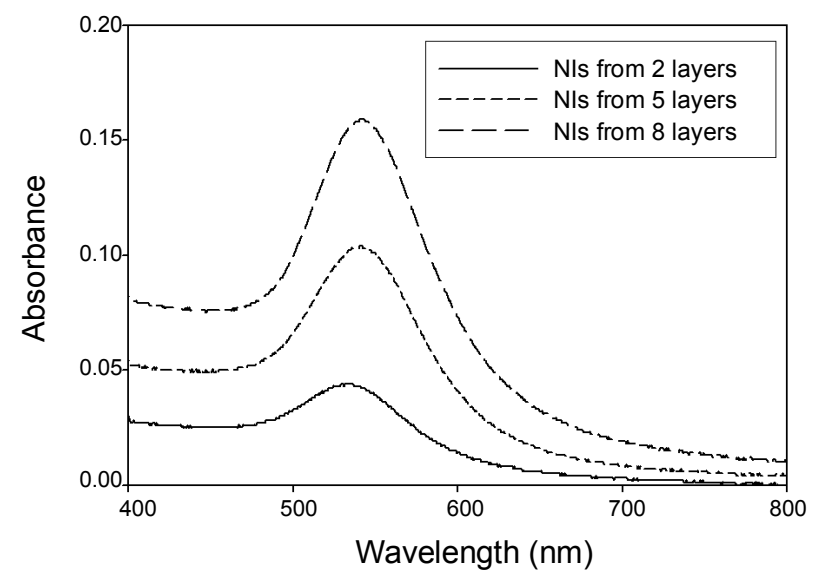

Figure 3. UV-vis spectra of gold nanoisland film arrays generated from 2-, 5-, and 8-layer nanoparticle assemblies after annealing at $500{ }^{\circ} \mathrm{C}$ for 1 hour.

after the deposition on solid surfaces. ${ }^{35}$

The Au NPs-PAH films with 5-nanoparticle layers were each heated at $100{ }^{\circ} \mathrm{C}, 200{ }^{\circ} \mathrm{C}, 300{ }^{\circ} \mathrm{C}, 400{ }^{\circ} \mathrm{C}$, and $500{ }^{\circ} \mathrm{C}$ for one hour to see the effects of annealing temperature. The SP band at $\sim 540 \mathrm{~nm}$ of the multilayer films underwent a large evolution at temperatures above $300{ }^{\circ} \mathrm{C}$ (Figure 2). This spectral evolution suggests that the gold nanoparticles undergo nucleation and coalescence on the glass surface resulting in the formation of large grain-like structures. The absence of red-shift in the SP band after annealing indicates the annealed films formed single layers of laterally-spaced islands. Thin gold metallic films, which should exhibit a damped SP optical feature, were clearly not produced in any experiments performed here. ${ }^{32,41}$ The spectroscopic characteristics of films after annealing at $400{ }^{\circ} \mathrm{C}$ and $500{ }^{\circ} \mathrm{C}$ were almost identical. This indicates that the gold nanoisland-type structure was formed at the temperature above $400{ }^{\circ} \mathrm{C}$.

Formation and Characterization of Gold Nanoisland Films. It is known that heating treatments of relatively thick nanoparticle films at temperatures above $350^{\circ} \mathrm{C}$ resulted in the removal of organics (alkanethiols) and the formation of bare gold films on solid substrates. ${ }^{32}$ However, for the formation of gold nanoisland films (Scheme 1) in our approach, nanoparticle-polymer multilayer films were annealed at higher temperatures $\left(\sim 500^{\circ} \mathrm{C}\right)$ to thermally evaporate ionic polymers (PAH). Annealing of the films at $\sim 500^{\circ} \mathrm{C}$ was necessary to ensure the removal of PAH, which can be completely vaporized at $\sim 460^{\circ} \mathrm{C}$ based on the thermogravimetric analysis (TGA) result.
Table 1. Contact Angles of Water for Nanoparticle Multilayer and SAM-modified Nanoisland Films. ${ }^{a}$

\begin{tabular}{lc}
\hline \multicolumn{1}{c}{ Films } & $\begin{array}{c}\text { Contact Angles } \\
\left(\theta,{ }^{\circ}\right)\end{array}$ \\
\hline Au NPs-PAH multilayer films & $81 \pm 3$ \\
Au NPs-PAH multilayer films (annealed at $\left.200{ }^{\circ} \mathrm{C}\right)$ & $42 \pm 3$ \\
Au NPs-PAH multilayer films (annealed at $\left.400{ }^{\circ} \mathrm{C}\right)$ & $21 \pm 4$ \\
Au NPs-PAH multilayer films (annealed at $\left.500{ }^{\circ} \mathrm{C}\right) ;$ & $\sim 0$ \\
nanoisland films & $48 \pm 5$ \\
$\mathrm{CH}_{3}\left(\mathrm{CH}_{2}\right)_{5} \mathrm{SH}-$ modified gold nanoisland films & $43 \pm 4$ \\
$\mathrm{CH}_{3}\left(\mathrm{CH}_{2}\right)_{8} \mathrm{SH}-$ modified gold nanoisland films & $59 \pm 4$ \\
$\mathrm{CH}_{3}\left(\mathrm{CH}_{2}\right)_{11} \mathrm{SH}-$ modified gold nanoisland films & $62 \pm 5$ \\
$\mathrm{CH}_{3}\left(\mathrm{CH}_{2}\right)_{14} \mathrm{SH}-$ modified gold nanoisland films & \\
\hline
\end{tabular}

${ }^{a}$ The Au NPs-PAH 5-nanoparticle layer films were used for these contact angle studies.

Different gold nanoisland films were generated from $\mathrm{Au}$ NPs-PAH multilayer films with two, five or eight layers of gold nanoparticles after annealing at $\sim 500^{\circ} \mathrm{C}$. The optical properties of the films were examined using transmission UV-vis spectroscopy (Figure 3), showing strong SP bands around 533, 541, and $542 \mathrm{~nm}$ for nanoisland films generated from Au NPs-PAH 2-, 5-, and 8-nanoparticle layer films, respectively. Similar SP bands have been observed from Rubinstein's vacuum evaporated films after annealing at $200-250{ }^{\circ} \mathrm{C} .{ }^{19,21}$ Their study showed that the increase in the optical intensity was observed upon increasing the nominal thickness of the evaporated island films. UV-vis spectra of our nanoisland films generated from Au NPs-PAH multilayer films with 2-, 5-, or 8-layers of gold nanoparticles also showed a strong dependence of the localized gold SP band intensity on the composition of gold island films. The average SP band intensity of island films increased with increasing number of nanoparticle layers in nanoparticle multilayer films.

The contact angle measurements were performed to confirm the removal of organic matters from the surface. The data in Table 1 show that the average contact angles of water are $81 \pm 3^{\circ}$ for the Au NPs-PAH 5-nanoparticle layer films. The contact angles decrease as the annealing temperature increases, attesting no measurable angle after annealing at $\sim 500^{\circ} \mathrm{C}$. The data $(\theta=$ $\sim 0^{\circ}$; not measurable) for the corresponding films after annealing at $\sim 500{ }^{\circ} \mathrm{C}$ suggest that the produced nanoisland films are quite hydrophilic. This phenomenon can plausibly arise from stronger interactions between water and the heterogeneous surfaces of gold nanoisland and exposed glass surfaces after thermal evaporation of organic matters from the films.

Since the reproducibility of the self-assembly/annealing method is very important, multiple batches of arrays with each batch containing more than 20 nanoisland films were prepared and their optical properties (intensity and wavelength of absorption (SP) bands) were examined. The results showed $>80 \%$ of produced nanoisland films have a similar average intensity of SP bands. The wavelength of the SP band $(541 \pm 4 \mathrm{~nm})$ was highly reproducible for all arrays produced. The $<20 \%$ of arrays, which have substantial deviation of SP band intensity, were discarded immediately and were not used for any further 

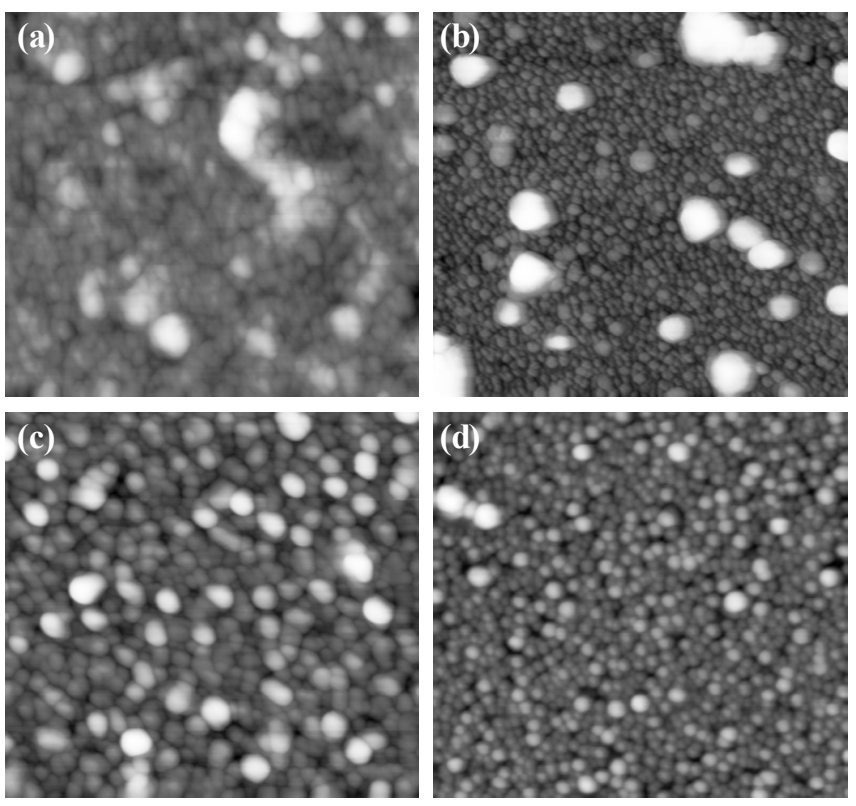

Figure 4. AFM images $(1 \mu \mathrm{m} \times 1 \mu \mathrm{m})$ of (a) nanoparticle multilayer (5nanoparticle layers) films (pre-heating), (b) nanoisland films generated from 2-layer nanoparticle films (post-heating at $500{ }^{\circ} \mathrm{C}$ ), (c) gold nanoisland films generated from 5-layer nanoparticle films (postheating at $500{ }^{\circ} \mathrm{C}$ ), and (d) gold nanoisland films generated from 8layer nanoparticle films (post-heating at $500{ }^{\circ} \mathrm{C}$ ).

experiment. The color of the defected arrays was noticeably dimmer (pink vs. purple), which made the defected slides easily distinguishable from the other arrays that were in a good condition. The formation of defected slides is likely driven by the poor quality of the glass substrates and the incomplete functionalization of glass surfaces with MPTS.

Atomic force microscopy (AFM) has been used as a good estimation tool for the shapes and sizes of the islands. Despite the AFM tip convolution effect, AFM images have been found to correspond well with high resolution scanning electron microscopy (HRSEM) results. ${ }^{21}$ The AFM image of Au NPs-PAH multilayer (5-nanoparticle layer) films was slightly inhomogeneous and a bit blurry, most likely due to the presence of soft polymer linkers and other organic matters (Figure 4.a). After annealing at $500{ }^{\circ} \mathrm{C}$ for one hour, the films showed rounded, nanoparticlelike morphologies with some separation among the islands in AFM images. These results confirmed that the annealing of $\mathrm{Au}$ NPs-PAH multilayer films at $\sim 500^{\circ} \mathrm{C}$ caused the nucleation and coalescence of gold nanoparticles and the formation of nanoisland structures on the glass surfaces (Figure 4.b-d).

AFM results also showed that, by changing the number of particle layers in Au NPs-PAH multilayer films, one can control the density and the average diameter of island grains. Gold nanoisland films obtained from 2-nanoparticle layer films (with one PAH layer) turned out to be quite polydisperse (Figure 4.b). There were many small grains all over the surface. However, several larger island structures were also observed. The overall concentration of gold nanoparticles in the Au NPs-PAH 2-nanoparticle layer films was most likely too low to produce monodisperse island structures. The small grain sizes for these nanoisland films generated from Au NPs-PAH 2-nanoparticle layer

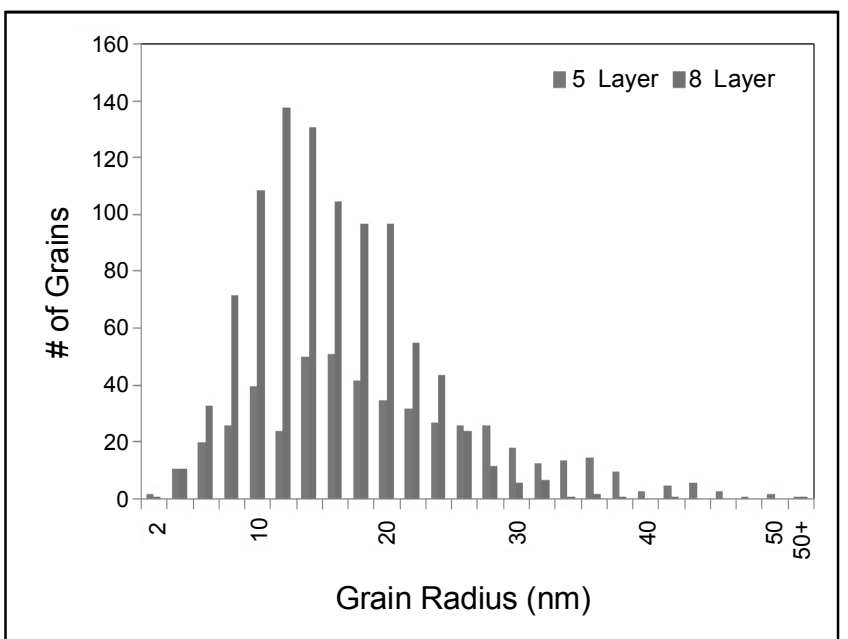

Figure 5. Size histograms of gold nanoisland films generated from 5and 8-nanoparticle layer films.

films was clearly reflected in the UV-vis spectrum (Figure 3), showing a lower intensity of gold SP band. The lower wavelength of the SP band at $533 \mathrm{~nm}$ compared to the SP bands (541-542 nm) of nanoisland films generated from 5-nanoparticle and 8-nanoparticle layer films also suggested that the grains in these films resemble large gold nanoparticles (SP bands at $\sim 520 \mathrm{~nm}$ ). Nanoisland films from 5-nanoparticle and 8-nanoparticle layer films were more monodispersed as shown in Figure 4.c and 4.d.

After modification of the AFM images to remove the scanning artifacts, the watershed algorithm in Image $\mathrm{J}$ was used to measure and analyze island grains. The grains in the edge of the scanned area were not included for the analysis to reduce the error. Figure 5 is a histogram showing the number of island grains against the radii for nanoisland films generated from $\mathrm{Au}$ NPs-PAH 5-nanoparticle layer and 8-nanoparticle layer films. The nanoisland films generated from Au NPs-PAH 2-nanoparticle layer films could not be analyzed because the analysis required the techniques beyond the scope of current algorithm. The average radius of the nanoisland films from 5-nanoparticle layer was $19.08 \pm 9.94 \mathrm{~nm}$ and that of nanoisland films from 8nanoparticle layer films was $14.43 \pm 5.91 \mathrm{~nm}$. The morphologies of these nanoisland film arrays were quite comparable to those of nanoisland films prepared by vacuum evaporation method. ${ }^{15-22}$ As shown in Figure 5, the nanoisland films generated from 8-nanoparticle layer films had more grains and the peak of the distribution moved to a lower value. Density of grains for nanoisland films from 5- and 8-nanoparticle layer films was estimated to be $\sim 500$ grains $/ \mu \mathrm{m}^{2}$ and $\sim 950$ grains $/ \mu^{2}$, respectively. These results showed that the gold nanoisland films generated from 8-nanoparticle layers have a much higher island density along with a high concentration of smaller grains. The high concentration of smaller island domains for nanoisland films generated from Au NPs-PAH 8-nanoparticle layer films was indicated in UV-vis spectrum (Figure 3) as highly intense SP bands of gold. The wavelength of the SP bands ( $541-542$ $\mathrm{nm}$ ) was almost identical for the nanoisland films generated from 5- and 8-nanoparticle layers. The similar wavelength of SP 
bands for two nanoisland films was likely resulted from the combination of different island density (concentration) and different average island size, canceling out the effects of each other.

These results were somewhat different with the trend observed by Rubinstein's vacuum evaporation method. ${ }^{19}$ Rubinstein showed that, in general, the average island size increases with the increased amount of evaporated gold. Despite the increased concentration of nanoparticles (from 5 layers to 8 layers; $60 \%$ increase) per area, the average size of nanoisland arrays generated from 8-nanoparticle layer films was even smaller. The concentration of polymer linkers (from 4 layers to 7 layers; $75 \%$ increase) in Au NPs-PAH 8-nanoparticle layer films was increased even more and seems to be an important factor for these results. The increased concentration of $\mathrm{PAH}$ linkers might have limited the nucleation of nanoparticles more during the annealing process of Au NPs-PAH 8-nanoparticle layer films, resulting in the formation of smaller grains.

Formation of Self-assembled Monolayers on Gold Nanoisland Films. Assembly of various thiol monolayers with different $\omega$-functional groups will be important for future specific and nonspecific binding studies using gold nanoisland films. In addition, self-assembled monolayers (SAMs) of alkanethiols provide an ideal system for studying surface properties of coated gold nanoisland arrays. ${ }^{43}$ In the technique of self assembly, the exposure of a gold surface to a solution of alkanethiol or dialkyl disulfide results in the formation of a well defined monolayer films with the sulfur atoms binding to the gold surface. In this study, the monolayers were formed by exposing the as-prepared gold nanoisland film arrays generated from 5-nanoparticle layer to solutions containing alkanethiols (1-hexanethiol, 1-nonanethiol, 1-dodecanethiol, or 1-pentadecanethiol) for at least 24 hours. The arrays were then characterized with the contact angle goniometer (Table 1) as a simple means of detecting surface functionalization. The results clearly showed that the assembly of alkanethiols on gold nanoisland films makes the surface less wetting. The water contact angle of monolayer-coated nanoisland film arrays also showed, in general, a slight increase as the length of the alkanethiol increases. The increased contact angles $\left(\sim 62^{\circ}\right)$ reveal more hydrophobic properties of the selfassembled monolayers on gold nanoisland films as the chain length of alkanethiols increases. This phenomenon can arise from at least two factors. First, interactions between water and the surface of gold might be greater at the shorter chain lengths. Second, the disorder in the films might be greater at the shorter chain lengths. The water contact angle values of SAM-coated nanoisland films are still much lower than those of flat gold films coated with alkanethiols, which have the values usually between $105^{\circ}$ and $115^{\circ} .44$ This is not a surprising result because the interfacial surfaces that make contact with water are consisted of not only SAM-modified gold island surfaces but also hydrophilic bare glass surfaces.

Since the localized SP absorption characteristics of the island films in UV-vis spectra is highly sensitive to the dielectric constant of the surrounding medium, alkanethiol absorption results in changes in the SP band of gold. However these results were not reproducible and sometimes involved red-shifts or a decrease in the intensity of SP bands, indicating the changes in morphology of gold nanoisland films after exposure to alkanethiols. (a)

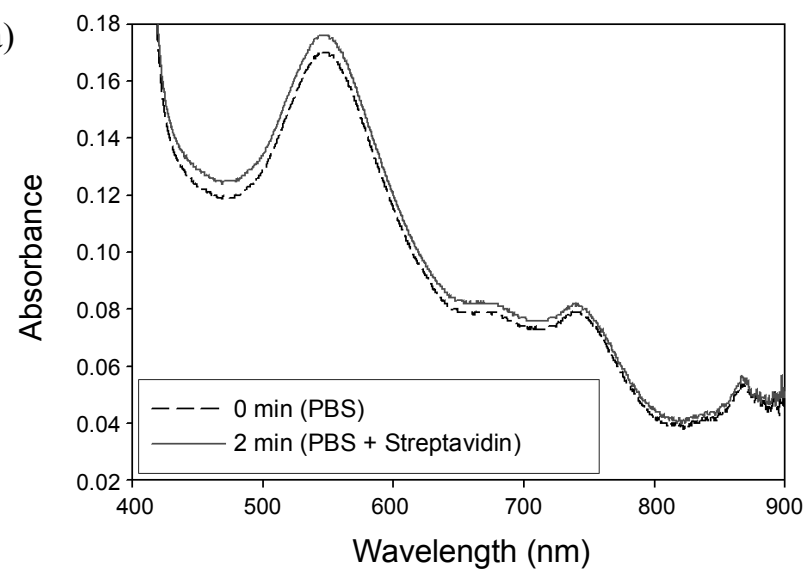

(b)

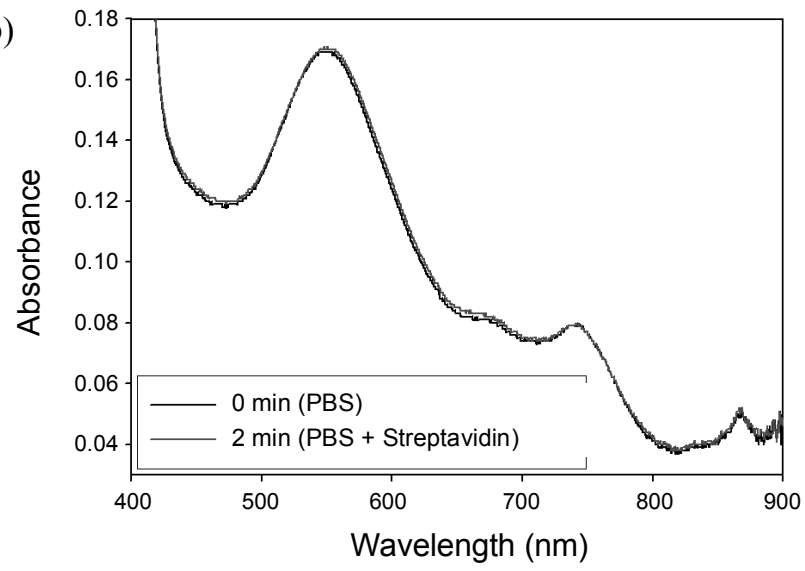

Figure 6. UV-vis spectra of biotin-functionalized gold nanoisland films prepared at $550^{\circ} \mathrm{C}$ before and after exposure to streptavidin with the concentrations of (a) $100 \mu \mathrm{g} / \mathrm{mL}$ and (b) $1 \mu \mathrm{g} / \mathrm{mL}$.

Glass slides are known to exhibit poor adhesion to noble metals. This instability of gold nanoisland films has also been observed from the nanoisland films prepared by the vacuum evaporation protocol. However, Rubinstein has recently reported methods (silica-coating or high-temperature post annealing) that can improve the overall stability of gold nanoisland films on glass substrates. ${ }^{19,45}$ Our study examining Rubinstein's high-temperature annealing also suggested the stability of nanoisland films dramatically improves after this simple treatment.

Interaction of Streptavidin with Biotin-Functionalized Gold Nanoisland Films. Strong interactions between biomolecules and functionalized nanoisland films will induce a change in the optical properties of the films, which can be monitored using UV-vis spectroscopy in transmission mode (T-LSPR). To briefly examine the sensing capability of gold nanoisland films annealed at $550{ }^{\circ} \mathrm{C}$, the biotin-modified nanoisland films were incubated in a solution of streptavidin $(100 \mu \mathrm{g} / \mathrm{mL}$ or $1 \mu \mathrm{g} / \mathrm{mL})$ in phosphate-buffered saline (PBS). ${ }^{46}$ For the real-time binding study, an extinction spectrum was acquired in $\mathrm{PBS}$ at $\mathrm{t}=0 \mathrm{~min}$, and then the PBS was replaced with the streptavidin solutions. Specific binding of streptavidin to biotin should result in the changes in T-LSPR band of nanoisland films. Our results showed that the intensity of SP band of gold was increased after the exposure of biotin-functionalized gold nanoisland films to 
streptavidin solution $(100 \mu \mathrm{g} / \mathrm{mL})$ in PBS for 2 min (Figure 6). ${ }^{47}$ No further changes were observed from the longer exposure of island films to streptavidin solution. In comparison, non-specific binding studies using MUA-functionalized gold nanoisland films showed no such change on the intensity of SP band of gold.

\section{Conclusion}

Gold nanoisland thin film arrays were prepared by thermal evaporation of organic matters from layer-by-layer assembled nanoparticle-polymer multilayer films. When the nanoparticle multilayer films were heat-treated, nucleation and coalescence of nanoparticles on the solid surface resulted in the formation of gold nanoisland arrays. This gold nanoisland film preparation method is simple and requires only basic laboratory supplies and equipment for the entire process. With ease in controlling the composition of nanoparticle multilayer films using the described self-assembly method, it was possible to develop nanoisland arrays with different average grain sizes. Specific binding of streptavidin to biotin-immobilized gold nanoisland films resulted in the increase in the intensity of SP band of gold. We are currently working on in-depth characterization of stabilized nanoisland films prepared in many different self-assembly conditions (nanoparticles with different sizes, annealing temperature, different polymer linkers, and number of nanoparticle layers) using SEM, AFM, and T-LSPR.

Acknowledgments. This research was supported from California State University Long Beach (Start-up and SCAC grants). We thank Thienloc Pham for his contribution during the Bridges to the Baccalaureate summer program at CSULB. MA thank Paul Gentry for his help on the grain distribution analysis.

\section{References}

1. Whang, D.; Jin, S.; Wu, Y.; Lieber, C. M. Nano Lett. 2003, 3, 12551259.

2. Röck, F.; Barsan, N.; Weimar, U. Chem. Rev. 2008, 108, 705-725.

3. Stewart, M. E.; Anderton, C. R.; Thompson, L. B.; Maria, J.; Gray, S. K.; Rogers, J. A.; Nuzzo, R. G. Chem. Rev. 2008, 108, 494-521.

4. Smith, E. A.; Corn, R. M. Applied Spectroscopy 2003, 57, 320A332A.

5. Lin, L.; Harris, J. W.; Thompson, H. G. R.; Brody, J. P. Anal. Chem. 2004, 76, 6555-6559.

6. Wark, A. W.; Lee, H. J.; Corn, R. M. Anal. Chem. 2005, 77, 39043907.

7. Yonzon, C. R.; Jeoung, E.; Zou, S.; Schatz, G. C.; Mrksich, M.; Van Duyne, R. P. J. Am. Chem. Soc. 2004, 126, 12669-12676.

8. Yonzon, C. R.; Stuart, D. A.; Zhang, X.; McFarland, A. D.; Haynes, C. L.; Van Duyne, R. P. Talanta 2005, 67, 438-448.

9. Zhao, J.; Das, A.; Zhang, X.; Schatz, G. C.; Sligar, S. G.; Van Duyne, R. P. J. Am. Chem. Soc. 2006, 128, 11004-11005.

10. Whitney, A. V.; Elam, J. W.; Zou, S.; Zinovev, A. V.; Stair, P. C.; Schatz, G. C.; Van Duyne, R. P. J. Phys. Chem. B 2005, 109, 20522-20528.

11. Hicks, E. M.; Lyandres, O.; Hall, W. P.; Zou, S.; Glucksberg, M. R.; Van Duyne, R. P. J. Phys. Chem. C 2007, 111, 4116-4124.

12. Haes, A. J.; Hall, W. P.; Chang, L.; Klein, W. L.; Van Duyne, R. P. Nano Lett. 2004, 4, 1029-1034.

13. Haes, A. J.; Van Duyne, R. P. J. Am. Chem. Soc. 2002, 124, 1059610604.
14. Haes, A. J.; Zou, S.; Schatz, G. C.; Van Duyne, R. P. J. Phys. Chem. B 2004, 108, 6961-6968.

15. Kalyuzhny, G.; Vaskevich, A.; Ashkenasy, G.; Shanzer, A.; Rubinstein, I. J. Phys. Chem. B 2000, 104, 8238-8244.

16. Kalyuzhny, G.; Schneeweiss, M. A.; Shanzer, A.; Vaskevich, A.; Rubinstein, I. J. Am. Chem. Soc. 2001, 123, 3177-3178.

17. Kalyuzhny, G.; Vaskevich, A.; Schneeweiss, M. A.; Rubinstein, I. Chem. Eur. J. 2002, 8, 3850-3857.

18. Lahav, M.; Vaskevich, A.; Rubinstein, I. Langmuir 2004, 20, 73657367.

19. Doron-Mor, I.; Barkay, Z.; Filip-Granit, N.; Vaskevich, A.; Rubinstein, I. Chem. Mater. 2004, 16, 3476-3483.

20. Doron-Mor, I.; Cohen, H.; Barkay, Z.; Shanzer, A.; Vaskevich, A.; Rubinstein, I. Chem. Eur. J. 2005, 11, 5555-5562.

21. Ruach-Nir, I.; Bendikov, T. A.; Doron-Mor, I.; Barkay, Z.; Vaskevich, A.; Rubinstein, I. J. Am. Chem. Soc. 2007, 129, 84-92.

22. Karakouz, T.; Vaskevich, A.; Rubinstein, I. J. Phys. Chem. B 2008, 112, 14530-14538.

23. Marinakos, S. M.; Chen, S.; Chilkoti, A. Anal. Chem. 2007, 79, 5278-5283.

24. Mitsuishi, M.; Koishikawa, Y.; Tanaka, H.; Sato, E.; Mikayama, T.; Matsui, J.; Miyashita, T. Langmuir 2007, 23, 7472-7474.

25. Lin, H.-Y.; Chen, C.-T.; Chen, Y.-C. Anal. Chem. 2006, 78, 68736878.

26. Frederix, F.; Friedt, J.-M.; Choi, K.-H.; Laureyn, W.; Campitelli, A.; Mondelaers, D.; Maes, G.; Borghs, G. Anal. Chem. 2003, 75, 6894-6900.

27. Dahlin, A.; Zäch, M.; Rindzevicius, T.; Käll, M.; Sutherland, D. S.; Höök, F.; J. Am. Chem. Soc. 2005, 127, 5043-5048.

28. Tokareva, I.; Minko, S.; Fendler, J. H.; Hutter, E. J. Am. Chem. Soc. 2004, 126, 15950-15951.

29. Gluodenis, M.; Manley, C.; Foss, Jr., C. A. Anal. Chem. 1999, 71, 4554-4558.

30. Szunerits, S.; Das, M. R.; Boukherroub, R. J. Phys. Chem. C 2008, 112, 8239-8243.

31. Hu, X.; Wang, T.; Dong, S. J. Coll. Interface Sci. 2007, 316, 947953.

32. Wuelfing, W. P.; Zamborini, F. P.; Templeton, A. C.; Wen, X.; Yoon, H.; Murray, R. W. Chem. Mater. 2001, 13, 87-95.

33. Daniel, M.-C.; Astruc, D. Chem. Rev. 2004, 104, 293.

34. Shon, Y.-S. Metal Nanoparticles Protected with Monolayers: Synthetic Methods. In Dekker Encyclopedia of Nanoscience and Nanotechnology; Schwarz, J. A., Ed.; Marcel Dekker: New York, 2004; pp 1-11.

35. Isaacs, S. R.; Choo, H.; Ko, W.-B.; Shon, Y.-S. Chem. Mater. 2006, 18, 107-114.

36. Hicks, J. F.; Shon, Y.-S.; Murray, R. W. Langmuir 2002, 18, 22882294.

37. Hao, E.; Lian, T. Chem. Mater. 2000, 12, 3392-3396.

38. Chan, E. W. L.; Lee, D.-C.; Ng, M.-K.; Wu, G.; Lee, K. Y. C.; Yu, L. A. J. Am. Chem. Soc. 2002, 124, 12238-12243.

39. Krasteva, N.; Krustev, R.; Yasuda, A.; Vossmeyer, T. Langmuir 2003, 19, 7754-7760.

40. Luo, J.; Maye, M. M.; Han, L.; Kariuki, N. N.; Jones, V. W.; Lin, Y.; Engelhard, M. H.; Zhong, C.-J. Langmuir 2004, 10, 4254-4260.

41. Luo, J.; Jones, V. W.; Han, L.; Maye, M. M.; Kariuki, N. N.; Zhong, C.-J. J. Phys. Chem. B 2004, 108, 9669-9677.

42. Shimizu, T.; Teranishi, T.; Hasegawa, S.; Miyake, M. J. Phys. Chem. B 2003, 107, 2719-2724.

43. Love, J. C.; Estroff, L. A.; Kriebel, J. K.; Nuzzo, R. G.; Whitesides, G. M. Chem. Rev. 2005, 105, 1103-1170.

44. Shon, Y.-S.; Lee, S.; Perry, S. S.; Lee, T. R. J. Am. Chem. Soc. 2000, 122, 1278-1281.

45. Karakouz, T.; Tesler, A. B.; Bendikov, T. A.; Vaskevich, A.; Rubinstein, I. Adv. Mater. 2008, 20, 3893-3899.

46. Riboh, J. C.; Haes, A. J.; McFarland, A. D.; Yonzon, C. R.; Van Duyne, R. P. J. Phys. Chem. B 2003, 107, 1772-1780.

47. Frederix, F.; Friedt, J.-M.; Choi, K.-H.; Laureyn, W.; Campitelli, A.; Mondelaers, D.; Maes, G.; Borghs, G. Anal. Chem. 2003, 75, 6894-6900. 